\title{
Impact of Geographic Location on Vitamin D Status and Bone Mineral Density
}

\author{
Kyung-Jin Yeum ${ }^{1}$, Byeng Chun Song ${ }^{1}$ and Nam-Seok Joo ${ }^{2, *}$ \\ 1 Division of Food Bioscience, College of Biomedical and Health Sciences, Konkuk University, \\ Chungju-si 27478, Korea; kyeum@kku.ac.kr (K.-J.Y.); bcsong@kku.ac.kr (B.C.S.) \\ 2 Department of Family Practice and Community Health, Ajou University School of Medicine, \\ Suwon-si 16499, Korea \\ * Correspondence: jchcmc@daum.net; Tel.: +82-31-219-5314; Fax: +82-31-219-5218
}

Academic Editors: Marlena Kruger and Hope Weiler

Received: 27 October 2015; Accepted: 22 January 2016; Published: 2 February 2016

\begin{abstract}
A significant decline of serum 25-hydroxyvitamin D concentration [25(OH)D] with increasing latitude has been reported only for Caucasians. To determine the association between serum $25(\mathrm{OH}) \mathrm{D}$ and geographic location and its impact on bone mineral density (BMD) in an Asian population, a total of 17,508 subjects ( 8910 men and 8598 women) from the 2008-2010 Korea National Health and Nutrition Examination Survey (KNHANES) were stratified into four age groups and analyzed for 25(OH)D and BMD according to geographic location (South, $33^{\circ} \mathrm{N}-35^{\circ} \mathrm{N}$; Middle, $36^{\circ} \mathrm{N}$; North, $\left.37^{\circ} \mathrm{N}-38^{\circ} \mathrm{N}\right)$. Mean 25(OH)D were 47.7 and $41.2 \mathrm{nmol} / \mathrm{L}$; calcium intake, 564.9 \& $442.3 \mathrm{mg} / \mathrm{d}$; femoral neck BMD, $0.829 \& 0.721 \mathrm{~g} / \mathrm{cm}^{2}$; and lumbar spine BMD, $0.960 \& 0.918 \mathrm{~g} / \mathrm{cm}^{2}$ for men and women, respectively. Both men and women living in the South had significantly higher $25(\mathrm{OH}) \mathrm{D}$ and femoral neck BMD for those $\geqslant 50$ years old. Lumbar spine BMD was significantly higher in men $\geqslant 50$ years old, and for women 10-29 \& 50-69 years old living in the South. A 1 or 2 degree difference in latitude has a significant effect on serum 25(OH)D and BMD in this low vitamin D status population. Thus, consideration of geographic location for a recommendation of vitamin D intake may be necessary.
\end{abstract}

Keywords: bone mineral density; vitamin D status; geographic location; serum 25-hydroxyvitamin D

\section{Introduction}

The skin production of vitamin $\mathrm{D}_{3}$ from 7-dehydrocholesterol by ultraviolet $\mathrm{B}$ (UVB) exposure is well known [1]. The photosynthesis of vitamin $\mathrm{D}_{3}$ can be particularly important for a population with inadequate vitamin D status. Widespread vitamin D deficiency and insufficiency has been reported in many different areas of Asian countries including China [2], India [3], Japan [4] and Korea [5]. Considering limited food sources for vitamin D such as fatty fish [6], solar exposure can be one of the major factors contributing to vitamin D status in these populations. Undoubtedly, the role of vitamin $\mathrm{D}$ for bone health [7] can be pivotal. Although the Institute of Medicine (IOM) report indicated no beneficial effect of a serum 25-hydroxyvitamin $\mathrm{D}$ [25(OH)D] concentration above $50 \mathrm{nmol} / \mathrm{L}$ [8], their findings may not be relevant for these populations. In addition, calcium deficiency in Asians such as Chinese [9], Indians [10], Japanese [4], and Koreans [11-13] emphasizes the importance of adequate vitamin D status for proper bone mass development and maintenance.

The importance of the angle of sunlight splitting 7-dehydrocholesterol in the skin is well documented $[1,14,15]$. Notably, no vitamin D was reported to be synthesized between November and March in Boston $\left(42^{\circ} \mathrm{N}\right)$ for residents [14], and vitamin $\mathrm{D}_{3}$ production in the skin is reported to be minimal in winter at latitudes above $35^{\circ}$ [1]. In another study, it has been reported that solar 
exposure of 3.5 and $22.4 \mathrm{~min}$ in July and winter, respectively in Tsukuba $\left(36^{\circ} \mathrm{N}\right)$, and $76.4 \mathrm{~min}$ in winter in Sapporo $\left(43^{\circ} \mathrm{N}\right)$, were required for synthesis of $5.5 \mu \mathrm{g}$ vitamin $\mathrm{D}_{3}$ production in the face and the back of hands under a cloudless sky at noon. Furthermore, extended solar exposure time of $106 \mathrm{~min}$ and $271 \mathrm{~min}$ were estimated to be required at 9:00 a.m. and 3:00 p.m. in the same region [16] to synthesize the same amount. Nevertheless, an ecologic meta-regression analysis indicated that there was no overall influence of latitude for vitamin D status in Caucasians with lighter skin as well as non-Caucasians with darker skin [17]. In this study, the mean serum 25(OH)D concentration was $54 \mathrm{nmol} / \mathrm{L}$, which is considerably higher than the vitamin D status in many Asian countries. The impact of geographic location on vitamin D status and bone mass in an Asian population with low vitamin D status is unknown. Thus, it is important to understand the contributions of geographic location to vitamin $\mathrm{D}$ status and bone mass in population with widespread vitamin D deficiency in order to target interventions most effectively. Therefore, we aimed to evaluate the importance of geographic location on vitamin D status and bone mineral density using data from the fourth and fifth Korea National Health and Nutrition Examination Survey (KNHANES).

\section{Methods and Materials}

\subsection{Study Population}

The Korean National Health and Nutrition Examination Survey (KNHANES), conducted periodically by the Korea Centers for Disease Control and Prevention since 1998, provides comprehensive information on health status, health behavior, nutritional status, and socio-demographics in national districts in Korea. The fourth (IV-2 and IV-3, 2008, 2009) and fifth (V-1, 2010) KNHANES data containing serum 25(OH)D concentration and Dual X-ray Energy Absorptiometry (DXA) were used in this cross-sectional analysis. From an initial total of 29,235 males and females, 11,727 subjects (missing data of serum $25(\mathrm{OH}) \mathrm{D}$, current cancers, any supplement user, oral contraceptive users, hormone replacement therapy) were excluded. Of 17,508 evaluated subjects, 3665 subjects were also excluded for missing data of DXA. A final 13,843 participants (7064 males and 6779 females) were used in this analysis as shown in Figure 1. All participants provided written informed consent before taking the survey.

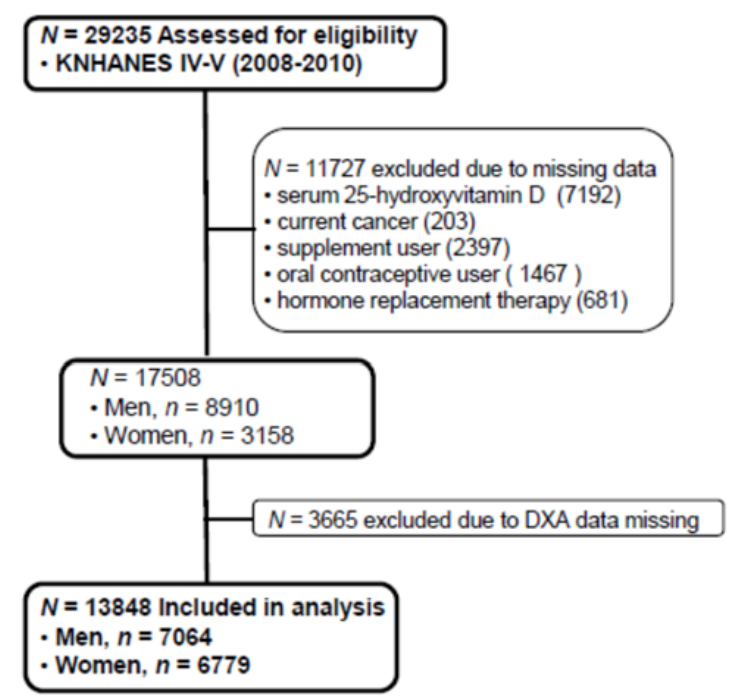

Figure 1. Flow diagram of subject inclusion and exclusion in the Korea National Health and Nutrition Examination Surveys (KNHANES IV \& V). DXA: Dual energy X-ray absorptiometry.

\subsection{Laboratory and Nutritional Assessment}

Blood samples were collected year-round after an 8-h fasting. Samples were immediately processed, refrigerated, and transported via cold storage to the central testing institute (NeoDin 
Medical Institute, Seoul, South Korea) where they were then analyzed within $24 \mathrm{~h}$. Serum 25(OH)D concentration was measured with a radioimmunoassay (RIA) kit (DiaSorin Inc., Stillwater, MN, USA) using an r-counter (1470Wizard; PerkinElmer, Turku, Finland). The inter-assay coefficients of variation (CV) were $2.8 \%-6.2 \%$ for samples in 2008-2009 and 1.9\%-6.1\% for samples in 2010. Serum 25(OH)D was measured in the same institute, which conducted quality control measures every other week throughout the analysis period in order to minimize analytical variation. Bone mineral density (BMD) $\left(\mathrm{g} / \mathrm{cm}^{2}\right)$ of the lumbar spine (L1-4) and femoral neck was measured by dual-energy X-ray absorptiometry (DISCOVERY-W fan-beam densitometer, Hologic Inc., Bedford, MA, USA) with CVs of $1.9 \%$ and $2.5 \%$, respectively. Nutrient intakes, including total calorie and calcium intakes, were assessed with a 24-h dietary recall questionnaire administered by a trained dietician. The results were calculated using the Food Composition Table developed by the National Rural Resources Development Institute (7th revision).

\subsection{Lifestyle Questionnaires}

Physical activity was assessed by a questionnaire and categorized as "yes" or "no"; "yes" meaning $>30$ min of moderate physical activity for three or more times in the last week. Current smokers were defined as those who were currently smoking and had smoked more than five packs of cigarettes during their life; ex-smokers were persons who had smoked in the past but had quit; nonsmokers had no history of smoking. Regular alcohol drinkers were those who drank alcohol more than one time per month, and nondrinkers were all others. Occupations were classified into two groups: indoor workers and outdoor workers. Indoor workers included managers, professionals and related workers, clerical office workers, service workers, and sales workers. Unemployed subjects were classified as indoor workers considering their limited outdoor activities. Outdoor workers included skilled agricultural, forestry, and fishery workers.

\subsection{Statistical Analysis}

The Korea Centers for Disease Control and Prevention recommended using the KNHANES data by complex sample analysis through proper data weighting. The data of males and females were divided into age groups (10 to 29,30 to 49,50 to 69 , and $>70$-years-old) and the latitude in which they lived in. The latitudes of South Korea ranges from $33^{\circ}$ to $38^{\circ}$ and the KNHANES data were composed of city unit. Therefore, we divided geographical locations into three different regions, South $\left(33^{\circ}\right.$ to $35^{\circ}$ ), Middle $\left(36^{\circ}\right)$ and North $\left(37^{\circ}\right.$ to $\left.38^{\circ}\right)$. General characteristics including age, body weight, body mass index (BMI), fat mass (measured by Dual Energy X-ray Absorptiometry), daily calcium intakes, serum $25(\mathrm{OH}) \mathrm{D}$, femur neck, and lumbar spine BMD were evaluated after weighing all values without any adjustment using a general linear model. In addition, smoking, drinking, and moderate physical activity was evaluated by a $\chi^{2}$ test in both genders. We compared serum $25(\mathrm{OH}) \mathrm{D}$ concentration and BMD (total hip, femur neck, and lumbar spine) according to each age group (10 to 29, 30 to 49, 50 to 69 , and $>70$-years-old) and region (South, Middle, North) by ANCOVA test after adjustments for variables affecting serum $25(\mathrm{OH}) \mathrm{D}$ concentrations. These variables were total body fat, smoking status, alcohol intake, moderate activity and education for men; total body fat, smoking status, alcohol intake, moderate activity, education, menopause, oral contraceptive use, and hormone replacement therapy for women. All $p$-values were $p$ for trend to assess the significance of all analyses and $p<0.05$ was considered significant. Data were analyzed using SPSS 19.0 (SPSS Inc., Chicago, IL, USA).

\section{Results}

\subsection{General Characteristics of Study Subjects Categorized by Region and Age}

General characteristics of men and women by regions are presented in Table 1. Mean calcium intakes for men were $570.8,550.8$ and $567.9 \mathrm{mg} / \mathrm{d}$, and for women were $448.0,400.3$ and $448.7 \mathrm{mg} / \mathrm{d}$ in the North, Middle and South, respectively. Mean serum 25(OH)D for men were 46.0, 52.7 and 
$51.1 \mathrm{nmol} / \mathrm{L}(p<0.001)$ in the North, Middle and South. Male fat mass was highest in the North $(16.1 \mathrm{~kg})$, followed by the Middle $(15.5 \mathrm{~kg})$, and the lowest in the South $(14.8 \mathrm{~kg})$. The mean serum 25(OH)D for women were 39.7, 44.1 and $44.6 \mathrm{nmol} / \mathrm{L}(p<0.001)$ in the North, Middle and South, respectively. There was no significant difference in fat mass with respect to regions in women (18.9, 18.8 and $18.4 \mathrm{~kg}$ for the North, Middle and South). The serum 25(OH)D sampling was evenly distributed across the four seasons since KNHANES was conducted year-round. Men living in the North, Middle and South had mean femoral neck bone mineral density (BMD) of $0.834,0.808$ and $0.827 \mathrm{~g} / \mathrm{cm}^{2}$, respectively, and Lumbar spine BMD of $0.959,0.949$ and $0.963 \mathrm{~g} / \mathrm{cm}^{2}$, respectively. Women living in the North, Middle and South presented mean femoral neck bone mineral density (BMD) of 0.725, 0.706 and $0.720 \mathrm{~g} / \mathrm{cm}^{2}$, respectively, and Lumbar spine BMD of 0.922, 0.892 and $0.918 \mathrm{~g} / \mathrm{cm}^{2}$, respectively. Almost half of the subjects were living in the North (48.5\% and $46.9 \%$ men and women, respectively), followed by the South (39.8\% and $40.9 \%$ men and women, respectively), with the smallest number of subjects living in the Middle (11.7\% and $12.2 \%$ men and women, respectively).

Table 1. General characteristics of study subjects by regions and genders.

\begin{tabular}{|c|c|c|c|c|}
\hline \multicolumn{5}{|c|}{ Men $(n=7064)$} \\
\hline & North $\left(37^{\circ} \mathrm{N}\right)$ & Middle $\left(36^{\circ} \mathrm{N}\right)$ & South $\left(33^{\circ} \mathrm{N} \sim 36^{\circ} \mathrm{N}\right)$ & $p$ \\
\hline Numbers, $n(\%)$ & $3428(48.5)$ & $824(11.7)$ & $2811(39.8)$ & \\
\hline Age (years) & $39.9(0.4)$ & $44.0(1.0)$ & $41.3(0.6)$ & $<0.001$ \\
\hline Body weight (kg) & $69.9(0.3)$ & $69.0(0.7)$ & $68.0(0.3)$ & $<0.001$ \\
\hline BMI $\left(\mathrm{kg} / \mathrm{m}^{2}\right)$ & $24.0(0.1)$ & $24.0(0.2)$ & $23.5(0.1)$ & $<0.001$ \\
\hline Fat mass (kg) & $16.1(0.2)$ & $15.5(0.3)$ & $14.8(0.2)$ & $<0.001$ \\
\hline Calcium intake $(\mathrm{mg} / \mathrm{d})$ & $570.8(8.3)$ & $550.8(18.9)$ & $567.9(9.5)$ & 0.629 \\
\hline 25OHD (nmol/L) & $46.0(0.7)$ & $52.7(1.7)$ & $51.1(0.9)$ & $<0.001$ \\
\hline FNBMD $\left(\mathrm{g} / \mathrm{cm}^{2}\right)$ & $0.834(0.003)$ & $0.808(0.007)$ & $0.827(0.004)$ & 0.004 \\
\hline $\operatorname{LSBMD}\left(\mathrm{g} / \mathrm{cm}^{2}\right)$ & $0.959(0.003)$ & $0.949(0.007)$ & $0.963(0.004)$ & 0.244 \\
\hline \multicolumn{5}{|c|}{ Smoking ${ }^{*}, n\left(\%^{\dagger}\right)$} \\
\hline Current & $1336(44.1)$ & $312(41.5)$ & $1156(45.6)$ & $<0.001$ \\
\hline Past and never & $1696(55.9)$ & $440(58.5)$ & $1380(54.4)$ & \\
\hline \multicolumn{5}{|c|}{ Alcohol intake $*, n\left(\%^{\dagger}\right)$} \\
\hline Yes & $2282(75.7)$ & $552(73.5)$ & $1887(74.4)$ & $<0.001$ \\
\hline No & $733(24.3)$ & $199(26.5)$ & $648(25.6)$ & \\
\hline \multicolumn{5}{|c|}{ Physical activity ${ }^{*}, n\left(\%^{\dagger}\right)$} \\
\hline Yes & $329(10.9)$ & $146(19.4)$ & $375(14.8)$ & $<0.001$ \\
\hline No & $2701(89.1)$ & $606(80.6)$ & $2160(85.2)$ & \\
\hline \multicolumn{5}{|c|}{ Occupation ${ }^{*}, n\left(\%^{\dagger}\right)$} \\
\hline Indoor workers & $2904(84.7)$ & $687(83.4)$ & $2423(86.2)$ & $<0.001$ \\
\hline Outdoor workers & $243(7.1)$ & $87(10.6)$ & $192(6.8)$ & \\
\hline No jobs & $282(8.2)$ & $50(6.1)$ & $196(7.0)$ & \\
\hline \multicolumn{5}{|c|}{ Women $(n=6779)$} \\
\hline & North $\left(37^{\circ} \mathrm{N}\right)$ & Middle $\left(36^{\circ} \mathrm{N}\right)$ & South $\left(33^{\circ} \mathrm{N} \sim 36^{\circ} \mathrm{N}\right)$ & $p$ \\
\hline Numbers, $n(\%)$ & $3182(46.9)$ & $826(12.2)$ & $2771(40.9)$ & \\
\hline Age (years) & $37.0(0.4)$ & $42.6(1.5)$ & $42.5(0.7)$ & 0.001 \\
\hline Body weight (kg) & $56.5(0.2)$ & $56.4(0.3)$ & $56.5(0.2)$ & 0.975 \\
\hline $\mathrm{BMI}\left(\mathrm{kg} / \mathrm{m}^{2}\right)$ & $22.7(0.1)$ & $23.0(0.2)$ & $22.9(0.1)$ & 0.173 \\
\hline Fat mass (kg) & $18.9(0.2)$ & $18.8(0.3)$ & $18.4(0.2)$ & 0.079 \\
\hline Calcium intake $(\mathrm{mg} / \mathrm{d})$ & $448.0(6.7)$ & $400.3(10.4)$ & $448.7(8.9)$ & $<0.001$ \\
\hline $25 \mathrm{OHD}(\mathrm{nmol} / \mathrm{L})$ & $39.7(0.6)$ & $44.1(1.5)$ & $44.6(0.8)$ & $<0.001$ \\
\hline FNBMD $\left(\mathrm{g} / \mathrm{cm}^{2}\right)$ & $0.725(0.003)$ & $0.706(0.009)$ & $0.720(0.004)$ & 0.132 \\
\hline $\operatorname{LSBMD}\left(\mathrm{g} / \mathrm{cm}^{2}\right)$ & $0.922(0.004)$ & $0.892(0.010)$ & $0.918(0.004)$ & 0.029 \\
\hline
\end{tabular}


Table 1. Cont.

\begin{tabular}{|c|c|c|c|c|}
\hline \multicolumn{5}{|c|}{ Women $(n=6779)$} \\
\hline & North $\left(37^{\circ} \mathrm{N}\right)$ & Middle $\left(36^{\circ} \mathrm{N}\right)$ & South $\left(33^{\circ} \mathrm{N} \sim 36^{\circ} \mathrm{N}\right)$ & $p$ \\
\hline \multicolumn{5}{|c|}{ Smoking ${ }^{*}, n\left(\%^{\dagger}\right)$} \\
\hline Current & $196(6.8)$ & $43(5.8)$ & $108(4.3)$ & $<0.001$ \\
\hline Past and never & $2668(93.2)$ & $695(94.2)$ & $2420(95.7)$ & \\
\hline \multicolumn{5}{|c|}{ Alcohol intake ${ }^{*}, n\left(\%^{+}\right)$} \\
\hline Yes & $1178(41.4)$ & $261(35.4)$ & $945(37.4)$ & $<0.001$ \\
\hline No & $1670(58.6)$ & 477 (64.6) & $1583(62.6)$ & \\
\hline \multicolumn{5}{|c|}{ Physical activity ${ }^{*}, n\left(\%^{\dagger}\right)$} \\
\hline Yes & $254(8.9)$ & $178(24.2)$ & $384(15.2)$ & $<0.001$ \\
\hline No & $2610(91.1)$ & $559(75.8)$ & $2144(84.8)$ & \\
\hline \multicolumn{5}{|c|}{ Occupation ${ }^{*}, n\left(\%^{\dagger}\right)$} \\
\hline Indoor workers & $2737(86.0)$ & $695(84.1)$ & $2398(86.5)$ & $<0.001$ \\
\hline Outdoor workers & $252(7.9)$ & $79(9.6)$ & $208(7.5)$ & \\
\hline No jobs & $193(6.1)$ & $52(6.3)$ & $165(6.0)$ & \\
\hline
\end{tabular}

Data represent mean (SE, standard error) after weighing in complex sample analysis without adjustment. BMI: body mass index, Fat mass was measured by Dual-energy-X ray Absorptiometry (DXA); 25OHD: serum 25-hydroxyvitamin D concentration; FNBMD: femur neck bone mineral density; LSBMD: lumbar spine bone mineral density; ${ }^{*}$ missing data were not included; ${ }^{\dagger}$ percents of total study subjects in each region.

Table 2 presents the general characteristics of study subjects categorized by the four age groups. For both men and women, the lowest serum $25(\mathrm{OH}) \mathrm{D}$ concentration was in the 10 to 29 -year-old age group (42.7 and $38.9 \mathrm{nmol} / \mathrm{L})$. On the other hand, calcium intake was the lowest in the age group $\geqslant 70$-year-old for both men $(453.5 \mathrm{mg} / \mathrm{d})$ and women $(327 \mathrm{mg} / \mathrm{d})$.

Table 2. General characteristics of study subjects categorized by age.

\begin{tabular}{|c|c|c|c|c|c|}
\hline \multicolumn{6}{|c|}{ Age Groups of Men $(n=7064)$} \\
\hline & $10 \sim 29(n=1574)$ & $30 \sim 49(n=2548)$ & $50 \sim 69(n=2164)$ & $\geqslant 70(n=778)$ & $p$ \\
\hline Age (years) & $20.0(0.2)$ & $39.6(0.2)$ & $57.7(0.2)$ & $75.2(0.2)$ & \\
\hline Body weight (kg) & $66.1(0.4)$ & $71.9(0.3)$ & $67.7(0.3)$ & $61.4(0.4)$ & $<0.001$ \\
\hline BMI $\left(\mathrm{kg} / \mathrm{m}^{2}\right)$ & $22.5(0.1)$ & $24.4(0.1)$ & $24.1(0.1)$ & $22.8(0.1)$ & $<0.001$ \\
\hline Fat mass (kg) & $15.1(0.2)$ & $16.2(0.2)$ & $15.2(0.2)$ & $14.3(0.3)$ & $<0.001$ \\
\hline Calcium intake $(\mathrm{mg} / \mathrm{d})$ & $539.8(10.0)$ & $592.0(8.0)$ & $579.1(9.2)$ & 453.5 (14.5) & $<0.001$ \\
\hline $25 \mathrm{OHD}(\mathrm{nmol} / \mathrm{L})$ & $42.7(0.5)$ & $47.9(0.5)$ & $53.2(0.7)$ & $52.4(1.0)$ & $<0.001$ \\
\hline Physical activity, $n(\%)$ & $123(14.4)$ & $331(13.0)$ & $293(13.6)$ & $103(13.4)$ & 0.162 \\
\hline FNBMD $\left(\mathrm{g} / \mathrm{cm}^{2}\right)$ & $0.887(0.005)$ & $0.840(0.003)$ & $0.776(0.003)$ & $0.674(0.005)$ & $<0.001$ \\
\hline $\operatorname{LSBMD}\left(\mathrm{g} / \mathrm{cm}^{2}\right)$ & $0.939(0.006)$ & $0.983(0.003)$ & $0.955(0.004)$ & $0.915(0.008)$ & $<0.001$ \\
\hline \multicolumn{6}{|c|}{ Geography* $n(\%)$} \\
\hline North $\left(37^{\circ} \mathrm{N}\right)$ & $832(52.9)$ & $1296(50.9)$ & $976(45.1)$ & $325(41.8)$ & \\
\hline Middle $\left(36^{\circ} \mathrm{N}\right)$ & $151(9.6)$ & $267(10.5)$ & $286(13.2)$ & $120(15.4)$ & \\
\hline South $\left(33^{\circ} \mathrm{N} \sim 35^{\circ} \mathrm{N}\right)$ & $591(37.5)$ & $985(38.6)$ & $902(41.7)$ & $333(42.8)$ & \\
\hline \multicolumn{6}{|c|}{ Age Groups of Women $(n=6779)$} \\
\hline & $10 \sim 29(n=1554)$ & $30 \sim 49(n=2630)$ & $50 \sim 69(n=1747)$ & $\geqslant 70(n=848)$ & $p$ \\
\hline Age (years) & $19.7(0.2)$ & $39.6(0.1)$ & $57.8(0.2)$ & $76.1(0.2)$ & \\
\hline Body weight (kg) & $53.4(0.3)$ & $58.0(0.2)$ & $58.2(0.2)$ & $53.3(0.4)$ & $<0.001$ \\
\hline $\mathrm{BMI}\left(\mathrm{kg} / \mathrm{m}^{2}\right)$ & $20.9(0.1)$ & $23.0(0.1)$ & $24.5(0.1)$ & $24.0(0.1)$ & $<0.001$ \\
\hline Fat mass (kg) & $17.5(0.2)$ & $18.9(0.1)$ & $20.1(0.2)$ & $18.4(0.3)$ & $<0.001$ \\
\hline Calcium intake (mg/d) & $438.7(8.2)$ & $469.2(6.3)$ & $449.2(9.1)$ & $327.0(11.5)$ & $<0.001$ \\
\hline 25OHD (nmol/L) & $38.9(0.5)$ & $40.9(0.5)$ & $44.9(0.5)$ & $44.2(0.7)$ & $<0.001$ \\
\hline
\end{tabular}


Table 2. Cont.

\begin{tabular}{|c|c|c|c|c|c|}
\hline \multicolumn{6}{|c|}{ Age Groups of Women $(n=6779)$} \\
\hline & $10 \sim 29(n=1554)$ & $30 \sim 49(n=2630)$ & $50 \sim 69(n=1747)$ & $\geqslant 70(n=848)$ & $p$ \\
\hline Physical activity, $n(\%)$ & $92(9.8)$ & $325(12.4)$ & $304(17.6)$ & $95(11.3)$ & $<0.001$ \\
\hline $\operatorname{FNBMD}\left(\mathrm{g} / \mathrm{cm}^{2}\right)$ & $0.763(0.004)$ & $0.761(0.003)$ & $0.665(0.003)$ & $0.531(0.004)$ & $<0.001$ \\
\hline $\operatorname{LSBMD}\left(\mathrm{g} / \mathrm{cm}^{2}\right)$ & $0.917(0.004)$ & $0.996(0.003)$ & $0.843(0.004)$ & $0.730(0.006)$ & $<0.001$ \\
\hline \multicolumn{6}{|c|}{ Geography ${ }^{*}, n(\%)$} \\
\hline North $\left(37^{\circ} \mathrm{N}\right)$ & $806(51.9)$ & $1358(51.6)$ & $714(40.9)$ & $304(35.8)$ & \\
\hline Middle $\left(36^{\circ} \mathrm{N}\right)$ & $192(12.3)$ & $255(9.7)$ & $248(14.2)$ & $131(15.5)$ & \\
\hline South $\left(33^{\circ} \mathrm{N} \sim 36^{\circ} \mathrm{N}\right)$ & $556(35.8)$ & $1017(38.7)$ & $785(44.9)$ & $413(48.7)$ & \\
\hline
\end{tabular}

Data represent mean (SE). $p$ values are $p$ for trend from General linear model after data weighing in complex sample analysis without adjustment. BMI: body mass index; WC: waist circumference; Fat mass was measured by Dual-energy-X ray Absorptiometry (DXA); FNBMD: femur neck bone mineral density; LSBMD: lumbar spine bone mineral density. Physical activity: $>30$ min of moderate physical activity for three or more times in the last week. ${ }^{*} p$ values were from $\chi^{2}$ test.

Mean fat mass and BMI were highest in men of 30 to 49 -year-old $\left(16.2 \mathrm{~kg}\right.$ and $\left.24.4 \mathrm{~kg} / \mathrm{m}^{2}\right)$ and women 50 to 69 -year-old $\left(20.1 \mathrm{~kg}\right.$ and $\left.24.5 \mathrm{~kg} / \mathrm{m}^{2}\right)$ age groups. The femoral neck BMD was the highest in the 10 to 29-year-old age group in both men and women. With each progressively older age group, the femoral neck BMD of men declined to $94 \%, 87.5 \%$ and $76 \%$ at 30 to 49,50 to 69 and $\geqslant 70$-year-old age groups, respectively, and $99.7 \%, 87.2 \%$ and $70 \%$ at 30 to 49,50 to 69 and $\geqslant 70$-year-old age women, respectively; as compared to the 10 to 29 -year-old age group. Both men and women had the highest lumbar spine BMD in the 30 to 49 -year-old age group. The men in the $\geqslant 70$-year-old age group had the lumber spine BMD over $93 \%$ compared to the 30 to 49 -year-old age group men whereas women in the $\geqslant 70$-year-old age group declined to $73.3 \%$ compared to the 30 to 49 -year-old age group women.

\subsection{Serum 25(OH)D Concentrations Categorized by Geographic Location and Age}

The impact of geographic location on serum $25(\mathrm{OH}) \mathrm{D}$ concentration was examined by age group. The serum $25(\mathrm{OH}) \mathrm{D}$ concentrations were adjusted with potential confounders. The adjusted mean serum $25(\mathrm{OH}) \mathrm{D}$ concentrations by age and geographic location are shown in Figure 2. The serum $25(\mathrm{OH}) \mathrm{D}$ concentration in both men and women living in the South were significantly higher than those living in the North in all age groups except for men in the 10 to 29 year-old age group.

\subsection{Bone Mineral Density Categorized by Geographic Location and Age}

The impact of geographic location on bone mineral density was examined by age category after proper adjustment. The adjusted mean femoral neck BMD are shown in Figure 3. The femoral neck BMD for people living in the South was significantly higher than those of men and women living in the North at the 50 to 69 and $\geqslant 70$-year-old age groups.

In addition, men living in the South had significantly higher femoral neck BMD than those men living in the Middle at the $\geqslant 70$-year-old age. Women living in the south also had significantly higher femoral neck BMD than those women living in the Middle at the 60 to 69-yeal-old age group. As presented in Figure 4, those men living in the South had significantly higher lumbar spine BMD than those living in the North at the 50 to 69 and $\geqslant 70$-year-old age men groups as well as those living in the Middle at the $\geqslant 70$-year-old age men group. Those women living in the South also had significantly higher lumbar spine BMD than those living in the North at the 10 to 29 and 50 to 69-year-old age women groups. 


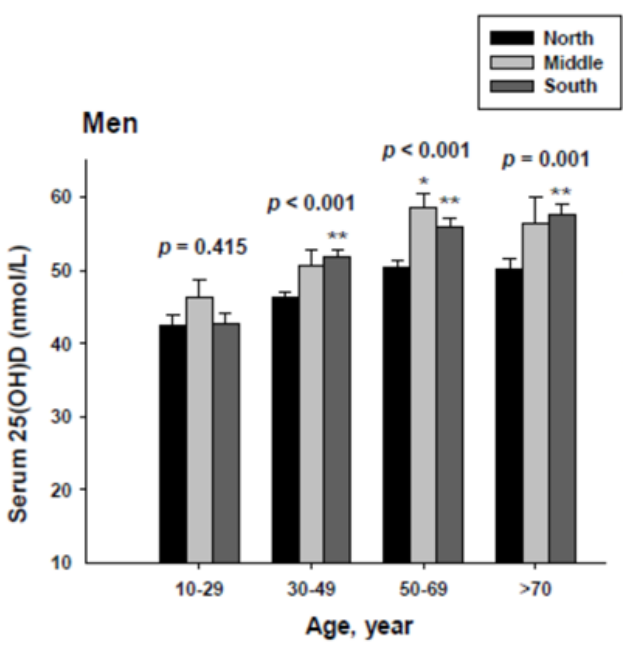

(A)

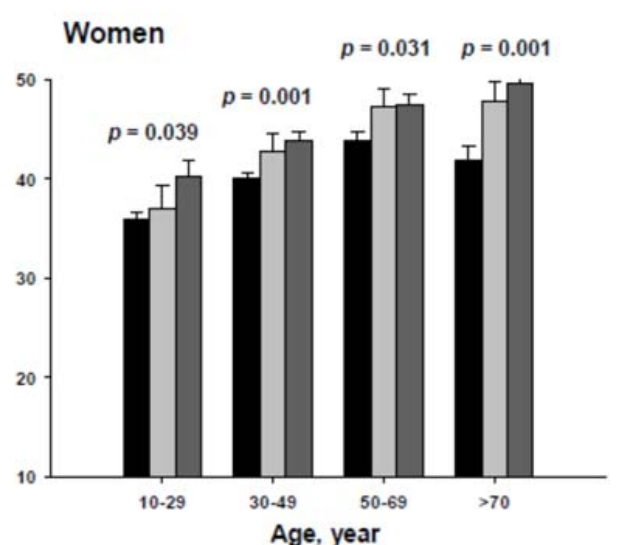

(B)

Figure 2. Adjusted mean serum $25(\mathrm{OH}) \mathrm{D}$ concentrations according to age and geographic location in men (A) and women (B). The $p$ values are $p$ for trend in the same group from ANCOVA test adjusted for total body fat, smoking, job, alcohol intake, moderate activity and education for men, and for total body fat, smoking, job, alcohol intake, moderate activity, education, menopause, oral contraceptive use and hormone replacement therapy for women Subject numbers for men are 10-29 years old, 832, 151,$591 ; 30-49$ years old, $1296,267,985 ; 50-69$ years old, 976, 286, 902; $\geqslant 70$ years old, 325, 120, 333 for North, Middle and South, respectively, and for women are 10-29 years old, 806, 192, 556; 30-49 years old, 1358, 255, 1017; 50-69 years old, 714, 248, 785; $\geqslant 70$ years old, 304, 131, 413 for North, Middle and South, respectively. ${ }^{*} p<0.05$, North $v$ s. Middle; ${ }^{* *} p<0.05$ North $v s$. South.

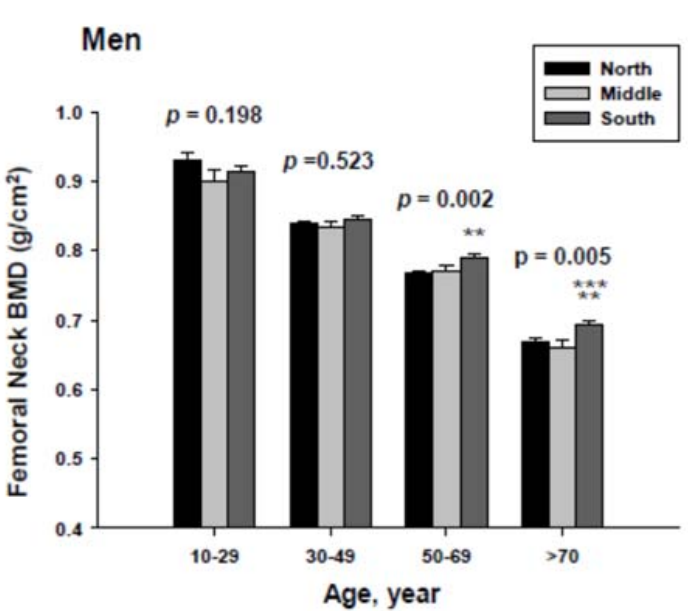

(A)

\section{Women}

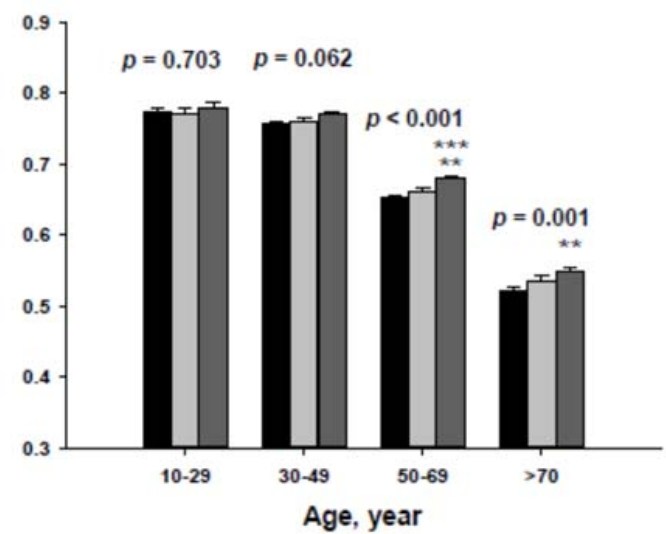

(B)

Figure 3. Adjusted mean femoral neck bone mineral densities according to age and geographic location in men (A) and women (B). The $p$ values are $p$ for trend in the same group from ANCOVA test adjusted for total body fat, smoking, job, alcohol intake, moderate activity and education for men, and for total body fat, smoking, job, alcohol intake, moderate activity, education, menopause, oral contraceptive use and hormone replacement therapy for women. Subject numbers for men are 10-29 years old, 832, 151,$591 ; 30-49$ years old, 1296, 267, 985; 50-69 years old, 976, 286, 902; $\geqslant 70$ years old, 325, 120, 333 for North, Middle and South, respectively, and for women are 10-29 years old, 806, 192, 556; 30-49 years old, 1358, 255, 1017; 50-69 years old, 714, 248, 785; $\geqslant 70$ years old, 304, 131, 413 for North, Middle and South, respectively.** $p<0.05$, North vs. South; ${ }^{* * *} p<0.05$ Middle vs. South. 


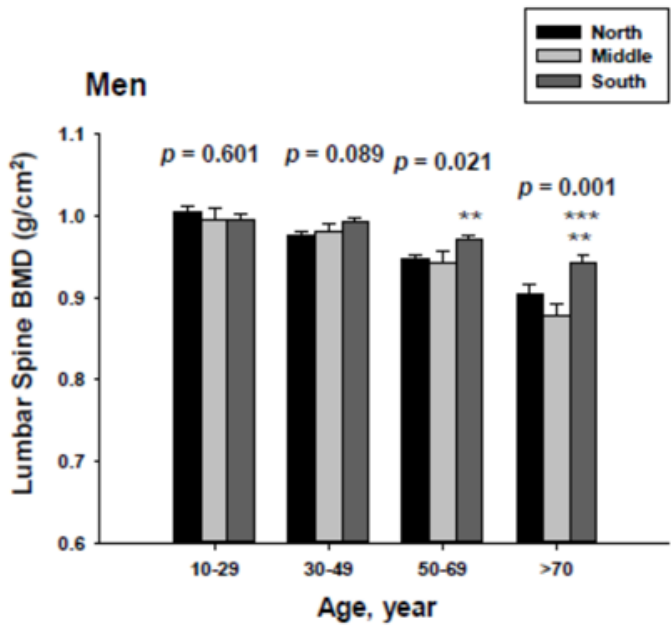

(A)
Women

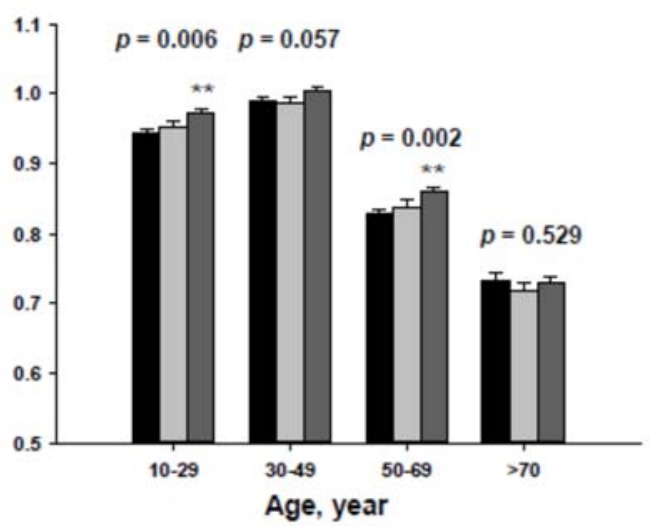

(B)

Figure 4. Adjusted lumbar spine bone mineral densities according to age and geographic location in men (A) and women (B).The $p$ values are $p$ for trend in the same group from ANCOVA test adjusted for total body fat, smoking, job, alcohol intake, moderate activity and education for men, and for total body fat, smoking, job, alcohol intake, moderate activity, education, menopause, oral contraceptive use and hormone replacement therapy for women. Subject numbers for men are 10-29 years old, 832, 151,$591 ; 30-49$ years old, 1296, 267, 985; 50-69 years old, 976, 286, 902; $\geqslant 70$ years old, 325, 120, 333 for North, Middle and South, respectively, and for women are 10-29 years old, 806, 192, 556; 30-49 years old, 1358, 255, 1017; 50-69 years old, 714, 248, 785; $\geqslant 70$ years old, 304, 131, 413 for North, Middle and South, respectively. ${ }^{* *} p<0.05$, North vs. South; ${ }^{* * *} p<0.05$ Middle vs. South.

\section{Discussion}

This study examined serum $25(\mathrm{OH}) \mathrm{D}$ concentration and BMD in relation to geographic location in a population with a high prevalence of low serum $25(\mathrm{OH}) \mathrm{D}$ and calcium intake. Due to the nature of this study, this cross-sectional study cannot establish causality. However, it does confirm that serum $25(\mathrm{OH}) \mathrm{D}$ concentration as well as BMD with a 1-2 degree difference in latitude can have a significant effect on vitamin D status. Although skin production of vitamin $\mathrm{D}_{3}$ by solar exposure is well established $[1,16]$, an ecologic meta-regression analysis reported no overall influence of latitude for vitamin D status [17]. In contrast, our study identified significant association between geographic location and vitamin D status. Furthermore, significant associations between geographic location and BMD were also found in our study. Unlike previous meta-analysis conducted in subjects with relatively adequate vitamin D status with mean serum $25(\mathrm{OH}) \mathrm{D}$ concentration of $54 \mathrm{nmol} / \mathrm{L}$, the current study population in Korea had mean serum 25(OH)D concentrations of 47.7 and $41.2 \mathrm{nmol} / \mathrm{L}$ for men and women, respectively. Notably, latitude has been reported to be a strong determinant of the relative contribution of different behavioral factors analyzed by a multiple regression model [18]. In addition, a recent study [19] indicated that location was one of the strongest factors associated with serum $25(\mathrm{OH}) \mathrm{D}$ concentrations $<75 \mathrm{nmol} / \mathrm{L}$ in subjects with human immunodeficiency virus. These findings emphasize that geographic location can be an important factor for vitamin D status in a population with vitamin D insufficiency.

Our findings indicate that subjects living in the South $\left(33^{\circ} \mathrm{N}\right.$ to $\left.35^{\circ} \mathrm{N}\right)$ had significantly higher serum 25(OH)D concentration as well as higher BMD of the femoral neck and lumbar spine than those living in the North $\left(37^{\circ} \mathrm{N}\right.$ to $\left.38^{\circ} \mathrm{N}\right)$. This implies that about $40 \%$ of the study population living in the North would benefit from increasing the duration of solar exposure as presented previously [16]. The current study subjects were divided into three different regions, South $\left(33^{\circ} \mathrm{N}-35^{\circ} \mathrm{N}\right)$, Middle $\left(36^{\circ} \mathrm{N}\right)$ and North $\left(37^{\circ} \mathrm{N}\right.$ to $\left.38^{\circ} \mathrm{N}\right)$, with consideration to minimal vitamin $\mathrm{D}_{3}$ production in the skin during the winter at latitudes above $35^{\circ}$ [1] as well as Asians' higher threshold for skin production 
of vitamin D compared to that of Caucasians [20]. This study indicates that there was no significant difference of serum $25(\mathrm{OH}) \mathrm{D}$ concentration according to geographic location for residents living above $35^{\circ} \mathrm{N}$. It is possible that there was minimal vitamin $\mathrm{D}_{3}$ production during the winter in the subjects living in the Middle and North. However, due to the small number of subjects living in the Middle, it is likely that this cohort does not have enough statistical power to show any significant difference.

It has been reported that ethnicity is a powerful modifier of the rate of BMD loss although body weight can also be a major determinant [21]. The annual rate of bone loss during the late perimenopause and the early post-menopause in the spine has been reported to be $1.8 \%-2.3 \%$ resulting in 7\%-10\% loss for 5 years [21]. As reported previously, Asian women had the most rapid BMD loss followed by Caucasian women, with the slowest BMD loss in African-American women. BMD loss of our study subjects were markedly accelerated during a menopause period resulting in $~ 30 \%$ difference in femoral neck and lumber spine BMD among different age groups in women. Although we do not know the optimal values of serum 25(OH)D and calcium intake for maintaining BMD in this population, it is clear that vitamin D status, which has been affected by the geographic location, plays a large role on bone loss.

Various factors are attributed to serum $25(\mathrm{OH}) \mathrm{D}$ concentrations such as skin synthesis of vitamin $\mathrm{D}_{3}$, intake of vitamin D-rich foods, and vitamin D-fortified foods. Considering the low intake of vitamin D-rich foods [22] and limited access to vitamin D-fortified foods [23] in this vitamin D insufficient population, this lends additional weight to the possibility of the potential impact of increased solar exposure on vitamin $\mathrm{D}$ status and consequent bone health. We were unable to assess the regional differences stratified by season due to the limitation of individual data access for both the season and region.

\section{Conclusions}

In conclusion, geographic location is a significant determinant of serum $25(\mathrm{OH}) \mathrm{D}$ concentration and BMD in this population with a high prevalence of low vitamin D and calcium status. This cross-sectional study suggests that a population living in any geographic location higher than $35^{\circ} \mathrm{N}$ may need to consider increasing of solar exposure or vitamin D intake. In contrast to previous findings in populations with an adequate vitamin D status, geographic location does impact overall vitamin D status and consequently bone health in this vitamin D insufficient population. A majority of the population in Korea living in a geographic location higher than $35^{\circ} \mathrm{N}$ are recommended to further increase solar exposure and/or vitamin D intake for improving bone mass in this at-risk population.

Acknowledgments: This work was supported by the faculty research fund of Konkuk University in 2013. The authors thank Jun-Goo Kwak at the University of Massachusetts Amherst for careful review of the manuscript.

Author contributions: Kyung-Jin Yeum and Nam-Seok Joo conceived and designed the experiments; Nam-Seok Joo performed the data analysis; Kyung-Jin Yeum, Byeng Chun Song, Nam-Seok Joo interpret the data, Kyung-Jin Yeum and Nam-Seok Joo draft the manuscript, Kyung-Jin Yeum, Byeng Chun Song and Nam-Seok Joo revised the manuscript and approved final version of manuscript.

Conflicts of interest: The authors declare no conflict of interest.

\section{References}

1. Holick, M.F.; Chen, T.C.; Lu, Z.; Sauter, E. Vitamin D and skin physiology: A D-lightful story. J. Bone Miner. Res. 2007, 22 (Suppl. 2), V28-V33. [CrossRef]

2. Lu, H.K.; Zhang, Z.; Ke, Y.H.; He, J.W.; Fu, W.Z.; Zhang, C.Q.; Zhang, Z.L. High prevalence of vitamin D insufficiency in China: Relationship with the levels of parathyroid hormone and markers of bone turnover. PLoS ONE 2012, 7, e47264. [CrossRef] [PubMed]

3. Ritu, D.; Gupta, A. Vitamin D deficiency in India: Prevalence, causalities and interventions. Nutrients 2014, 6, 729-775. 
4. Kirii, K.; Mizoue, T.; Iso, H.; Takahashi, Y.; Kato, M.; Inoue, M.; Noda, M.; Tsugane, S. Calcium, vitamin D and dairy intake in relation to type 2 diabetes risk in a Japanese cohort. Diabetologia 2009, 52, 2542-2550. [CrossRef] [PubMed]

5. Choi, H.S.; Oh, H.J.; Choi, H.; Choi, W.H.; Kim, J.G.; Kim, K.M.; Kim, K.J.; Rhee, Y.; Lim, S.K. Vitamin D insufficiency in Korea-A greater threat to younger generation: The Korea National Health and Nutrition Examination Survey (KNHANES) 2008. J. Clin. Endocrinol. Metab. 2011, 96, 643-651. [CrossRef] [PubMed]

6. Bendik, I.; Friedel, A.; Roos, F.F.; Weber, P.; Eggersdorfer, M. Vitamin D: A critical and essential micronutrient for human health. Front. Physiol. 2014, 5. [CrossRef] [PubMed]

7. Holick, M.F. Vitamin D deficiency. N. Engl. J. Med. 2007, 357, 266-281. [CrossRef] [PubMed]

8. Ross, A.C.; Manson, J.E.; Abrams, S.A.; Aloia, J.F.; Brannon, P.M.; Clinton, S.K.; Durazo-Arvizu, R.A.; Gallagher, J.C.; Gallo, R.L.; Jones, G.; et al. The 2011 report on dietary reference intakes for calcium and vitamin D from the Institute of Medicine: What clinicians need to know. J. Clin. Endocrinol. Metab. 2011, 96, 53-58. [CrossRef] [PubMed]

9. Villegas, R.; Gao, Y.T.; Dai, Q.; Yang, G.; Cai, H.; Li, H.; Zheng, W.; Shu, X.O. Dietary calcium and magnesium intakes and the risk of type 2 diabetes: The Shanghai Women's Health Study. Am. J. Clin. Nutr. 2009, 89, 1059-1067. [CrossRef] [PubMed]

10. Bhatia, V. Dietary calcium intake-A critical reappraisal. Indian J. Med. Res. 2008, 127, 269-273. [PubMed]

11. Joo, N.S.; Dawson-Hughes, B.; Kim, Y.S.; Oh, K.; Yeum, K.J. Impact of calcium and vitamin D insufficiencies on serum parathyroid hormone and bone mineral density: Analysis of the fourth and fifth Korea National Health and Nutrition Examination Survey (KNHAENS IV-3, 2009 and V-1, 2010). J. Bone Miner. Res. 2013, 28, 764-770. [CrossRef] [PubMed]

12. Joo, N.S.; Dawson-Hughes, B.; Yeum, K.J. 25-Hydroxyvitamin D, calcium intake, and bone mineral content in adolescents and young adults: Analysis of the fourth and fifth Korea National Health and Nutrition Examination Survey (KNHANES IV-2, 3, 2008-2009 and V-1, 2010). J. Clin. Endocrinol. Metab. 2013, 98, 3627-3636. [CrossRef] [PubMed]

13. Kim, K.H.; Lee, K.; Ko, Y.J.; Kim, S.J.; Oh, S.I.; Durrance, D.Y.; Yoo, D.; Park, S.M. Prevalence, awareness, and treatment of osteoporosis among Korean women: The Fourth Korea National Health and Nutrition Examination Survey. Bone 2012, 50, 1039-1047. [CrossRef] [PubMed]

14. Holick, M.F.; Siris, E.S.; Binkley, N.; Beard, M.K.; Khan, A.; Katzer, J.T.; Petruschke, R.A.; Chen, E.; de Papp, A.E. Prevalence of Vitamin D inadequacy among postmenopausal North American women receiving osteoporosis therapy. J. Clin. Endocrinol. Metab. 2005, 90, 3215-3224. [CrossRef] [PubMed]

15. Webb, A.R. Who, what, where and when-influences on cutaneous vitamin D synthesis. Prog. Biophys. Mol. Biol. 2006, 92, 17-25. [CrossRef] [PubMed]

16. Miyauchi, M.; Hirai, C.; Nakajima, H. The solar exposure time required for vitamin D3 synthesis in the human body estimated by numerical simulation and observation in Japan. J. Nutr. Sci. Vitaminol. 2013, 59, 257-263. [CrossRef] [PubMed]

17. Hagenau, T.; Vest, R.; Gissel, T.N.; Poulsen, C.S.; Erlandsen, M.; Mosekilde, L.; Vestergaard, P. Global vitamin $\mathrm{D}$ levels in relation to age, gender, skin pigmentation and latitude: An ecologic meta-regression analysis. Osteoporos. Int. 2009, 20, 133-140. [CrossRef] [PubMed]

18. Kimlin, M.G.; Lucas, R.M.; Harrison, S.L.; van der Mei, I.; Armstrong, B.K.; Whiteman, D.C.; Kricker, A.; Nowak, M.; Brodie, A.M.; Sun, J. The contributions of solar ultraviolet radiation exposure and other determinants to serum 25-hydroxyvitamin D concentrations in Australian adults: The AusD Study. Am. J. Epidemiol. 2014, 179, 864-874. [CrossRef] [PubMed]

19. Klassen, K.M.; Fairley, C.K.; Kimlin, M.G.; Kelly, M.; Read, T.R.; Broom, J.; Russell, D.B.; Ebeling, P.R. Ultraviolet index and location are important determinants of vitamin D status in people with human immunodeficiency virus. Photochem. Photobiol. 2015, 91, 431-437. [CrossRef] [PubMed]

20. Chen, T.C.; Chimeh, F.; Lu, Z.; Mathieu, J.; Person, K.S.; Zhang, A.; Kohn, N.; Martinello, S.; Berkowitz, R.; Holick, M.F. Factors that influence the cutaneous synthesis and dietary sources of vitamin, D. Arch. Biochem. Biophys. 2007, 460, 213-217. [CrossRef] [PubMed]

21. Finkelstein, J.S.; Brockwell, S.E.; Mehta, V.; Greendale, G.A.; Sowers, M.R.; Ettinger, B.; Lo, J.C.; Johnston, J.M.; Cauley, J.A.; Danielson, M.E.; et al. Bone mineral density changes during the menopause transition in a multiethnic cohort of women. J. Clin. Endocrinol. Metab. 2008, 93, 861-868. [CrossRef] [PubMed] 
22. Kwon, H.T.; Lee, C.M.; Park, J.H.; Ko, J.A.; Seong, E.J.; Park, M.S.; Cho, B. Milk intake and its association with metabolic syndrome in Korean: Analysis of the third Korea National Health and Nutrition Examination Survey (KNHANES III). J. Korean Med. Sci. 2010, 25, 1473-1479. [CrossRef] [PubMed]

23. Park, S.; Lee, B.K. Vitamin D deficiency is an independent risk factor for cardiovascular disease in Koreans aged $>/=50$ years: Results from the Korean National Health and Nutrition Examination Survey. Nutr. Res. Pract. 2012, 6, 162-168. [CrossRef] [PubMed] 